\title{
NIR-emitting benzene-fused oligo-BODIPYs for bioimaging
}

\author{
Gabriele Selvaggio, ${ }^{\mathrm{a}, \mathrm{b},{ }^{\dagger}}$ Robert Nißler, ${ }^{\mathrm{a}, \mathrm{b},{ }^{\dagger}}$ Peter Nietmann, ${ }^{\mathrm{b}}$ Atanu Patra, ${ }^{\mathrm{c}}$ Lukas J. Patalag, ${ }^{\mathrm{c}}$ \\ Andreas Janshoff, ${ }^{b}$ Daniel B. Werz ${ }^{c}$ and Sebastian Kruss ${ }^{a, b, d, *}$
}

Near-infrared (NIR) fluorophores are emerging tools for biophotonics because of their reduced scattering, increased tissue penetration and low phototoxicity. However, the library of NIR fluorophores is still limited. Here, we report the NIR fluorescence of two benzene-fused oligo-BODIPYs in their hexameric (H) and octameric (O) forms. These dyes emit bright NIR fluorescence (H: maxima 943/1075 nm, O: maxima 976/1115 nm) that can be excited in the NIR (H = 921 nm, $\mathrm{O}=956 \mathrm{~nm}$ ) or non-resonantly over a broad range in the visible region. The emission bands of $\mathrm{H}$ show a bathochromic shift and peak sharpening with increasing dye concentration suggesting the presence of J-aggregates. Furthermore, the emission maxima of both $\mathrm{H}$ and $\mathrm{O}$ shift up to $20 \mathrm{~nm}$ in solvents of different polarity. These dyes can be used as NIR ink and imaged remotely on the macroscopic level with a stand-off distance of $20 \mathrm{~cm}$. We furthermore demonstrate their versatility for biophotonics by coating microscale beads and performing microrheology via NIR video particle tracking (NIR-VPT) in biopolymer (F-actin) networks. No photodamaging of the actin filaments takes place, which is typically observed for visible fluorophores and highlights the advantages of these NIR dyes.

\section{Introduction}

Fluorophores emitting at near-infrared (NIR) wavelengths $(700 \mathrm{~nm}<\lambda<1700 \mathrm{~nm})$ have advantages compared to their counterparts in the visible range of the spectrum due to reduced scattering, photodamage, autofluorescence and absorption ${ }^{1,2}$. Such characteristics can be exploited especially for biological imaging, where high tissue penetration and high signal-to-noise ratios are desired ${ }^{1,3-6}$. However, up to now the number of available fluorophores is much smaller in the NIR compared to the visible. Furthermore, despite their high potential, most NIR fluorophores display lower quantum yields (QYs). There is therefore a high interest to design the "holy grail" of superior NIR fluorophores and pave the way for a broad range of novel and powerful tools for bioimaging.

Among the currently available NIR fluorophores, only two have so far been approved for clinical use: indocyanine green (ICG) and methylene blue (MB). Both are however emitting in the socalled NIR-I region (700 $\mathrm{nm}<\lambda<900 \mathrm{~nm}$ ), which, compared to regions of higher wavelengths (i.e. $\lambda>900 \mathrm{~nm}$ and the so-called NIR-II region with $1000 \mathrm{~nm}<\lambda<1700 \mathrm{~nm}$ ), present lower contrast and penetration depth in tissue ${ }^{1,7}$. For this reason, NIR-II emitters such as lanthanide-based nanocrystals, quantum dots (QDs), rare-earth-doped nanoparticles (RENPs), singlewalled carbon nanotubes (SWCNTs) and some organic dyes have received a lot of attention in recent years ${ }^{1,5,6}$. For instance, NIR fluorescent SWCNTs ${ }^{2}$ have become robust tools for targeted bioimaging and sensors applications $\mathrm{s}^{8,9}$ thanks to their fluorescence tunability ${ }^{10-12}$ in response to analytes of interest ${ }^{13,14}$ (e.g. neurotransmitters $\left.{ }^{15,16}\right)$, their established separation ${ }^{10,17}$ and functionalization routes ${ }^{8,18}$, as well as the possibility to perform defect chemistry ${ }^{19-21}$. 2D materials such as exfoliated silicate nanosheets (NS), on the other side, stand out for their high fluorescence brightness and remarkable photostability, which make them very promising labelling agents for multiple bioimaging scenarios 22,23 . Organic fluorophores tend to have shorter emission wavelengths and limited solubility in water but they also display faster excretion kinetics and can be chemically tailored, which makes them versatile materials for bioimaging ${ }^{6}$. Typical NIR dyes include canonical cyanines 24,25 (e.g. ICG), squaraines ${ }^{26,27}$, diketopyrrolopyrroles 28,29 , but also modified boron dipyrromethenes (BODIPYs) ${ }^{30,31}$. Structural modifications of the BODIPY core (e.g. aza-BODIPYs ${ }^{32,33}$, BOIMPYs ${ }^{34,35}$ ) are known to red-shift the spectral center of the parent BODIPY (around $500 \mathrm{~nm}$ ) up to the far-red and even NIR region. In this context, the straightforward lateral extension of the $\pi$-system ${ }^{36}$ (e.g. by Knoevenagel condensation ${ }^{37}$ ) is the most prominent strategy to tune the absorption and emission energies, resulting in appealing photophysical properties for e.g. biological labelling, photodynamic therapy, organic photovoltaics, etc. ${ }^{38,39}$ Recently, a novel three-step synthetic protocol to yield fully conjugated, benzene-fused oligo-BODIPYs from BODIPY precursors was presented ${ }^{38}$. With this procedure consisting in oligomerization and one-pot oxidation steps, a full series of oligomers ranging from dimers to octamers was synthesized. These chromophores displayed high stability and broad tunability of absorption and emission energies in the NIR region. While the absorption signatures were recorded for all oligoBODIPYs $^{38}\left(500 \mathrm{~nm}<\lambda_{\text {abs }}<950 \mathrm{~nm}\right)$, tetramers and larger molecules were reported to be non-fluorescent in the NIR region, presumably due to instrumental limitations.

Herein, we demonstrate the NIR fluorescence of an hexameric (H) and an octameric (O) oligo-BODIPY species. We characterize their NIR fluorescence via $1 \mathrm{D}$ and 2D NIR-fluorescence spectroscopy, and outline their performance in remote NIR imaging as well as in NIR particle tracking within biological cytoskeleton networks.

\footnotetext{
a. Department of Chemistry, Ruhr-University Bochum, 44801 Bochum, Germany.

Email: Sebastian.Kruss@rub.de

b. Institute of Physical Chemistry, Georg-August University Göttingen, 37077

Göttingen, Germany.

Technical University of Braunschweig, Institute of Organic Chemistry, 38106

Braunschweig, Germany.

d. Fraunhofer Institute for Microelectronic Circuits and Systems, 47057 Duisburg,

Germany.

† These authors contributed equally.

Electronic Supplementary Information (ESI) available.
} 


\section{Results and discussion}

The hexamer $(\mathrm{H})$ and the octamer $(\mathrm{O})$ form of benzene-fused oligo-BODIPYs present a large, arc-shaped structure which displays enhanced air stability and robustness against reversible reduction and oxidation cycles $^{38}$ (Figure 1a). Their main absorption and emission bands in dichloromethane (DCM) are centered at 921/943 nm (H) and 956/976 nm (O) (Figure 1b). Both absorption and emission stretch over a significantly large range from the visible to the NIR region (Figure S1), however the main bands are narrow, presenting full widths at half maximum (FWHM) between 650 and $800 \mathrm{~cm}^{-1}$ (Table 1). Interestingly, the dye concentration changed the ratio between the two largest emission peaks. Furthermore, bathochromic shifts are observed with increasing concentrations. The main emission band exhibits a red-shift of $25 \mathrm{~nm}(\mathrm{H})$ and $28 \mathrm{~nm}(\mathrm{O})$, whereas a red-shift of $6 \mathrm{~nm}$ of the second band of $\mathrm{H}$ was detected (Tables S1-S2, Figure S2a-b) ${ }^{39}$. These bathochromic shifts, together with the observed sharpening of the emission peaks in the case of the $\mathrm{H}$ dye, suggest the presence of $\mathrm{J}$ aggregates. Next to that, emission energies shifted with solvent polarity (Table S3, Figure S2c-d) ${ }^{38}$. We anticipate that the previously not reported NIR fluorescence of these oligoBODIPYs was due to low detection efficiencies of standard spectrometers in the $\mathrm{NIR}^{38}$. In contrast, we used equipment optimized for the NIR range (> $800 \mathrm{~nm}$, see Materials and Methods for details). a

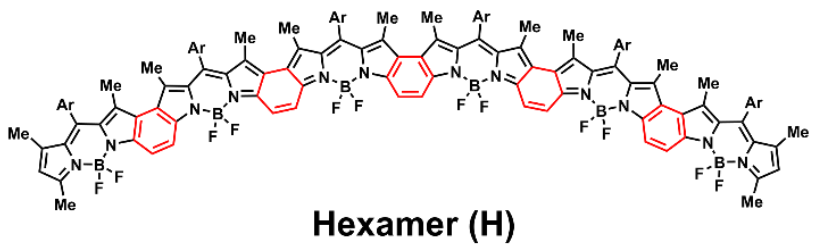

b

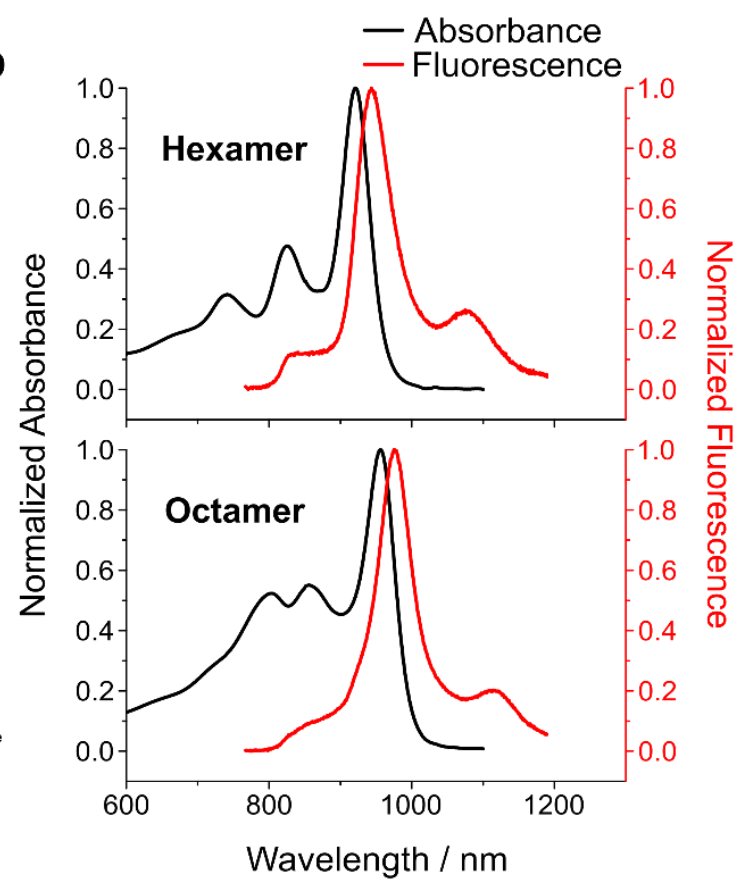

Figure 1: NIR fluorescent organic BODIPY dyes. a Chemical structure of the hexamer and octamer of benzene-fused oligo-BODIPYs. b Visible (Vis)-near-infrared (NIR) absorbance and NIR fluorescence spectra of the hexamer- and octamer-BODIPYs in dichloromethane (DCM).

Table 1: Absorption and emission features of hexameric $(\mathrm{H})$ and octameric $(\mathrm{O})$ benzene-fused oligo-BODIPYs in dichloromethane (DCM). The main absorption band $\left(\lambda_{\mathrm{abs}}\right)$, the first and second emission peaks $\left(\lambda_{\mathrm{em}, 1}\right.$ and $\left.\lambda_{\mathrm{em}, 2}\right)$, the full widths at half maximum of the mentioned signals $\left(\mathrm{FWHM}_{\mathrm{abs}}, \mathrm{FWHM}_{\mathrm{em}, 1}\right.$ and FWHM $\left.\mathrm{FWm}_{2}\right)$ and the Stokes shifts $(\Delta \tilde{\nu})$ are reported.

\begin{tabular}{|c|c|c|c|c|c|c|c|}
\hline Sample & $\lambda_{\text {abs }} / \mathrm{nm}\left(\mathrm{cm}^{-1}\right)$ & $\mathrm{FWHM}_{\mathrm{abs}} / \mathrm{nm}\left(\mathrm{cm}^{-1}\right)$ & $\lambda_{\mathrm{em}, 1} / \mathrm{nm}\left(\mathrm{cm}^{-1}\right)$ & $\mathrm{FWHM}_{\mathrm{em}, 1} / \mathrm{nm}\left(\mathrm{cm}^{-1}\right)$ & $\lambda_{\mathrm{em}, 2} / \mathrm{nm}\left(\mathrm{cm}^{-1}\right)$ & $\mathrm{FWHM} \mathrm{em}, 2_{2} / \mathrm{nm}\left(\mathrm{cm}^{-1}\right)$ & $\Delta \tilde{v} / \mathrm{nm}\left(\mathrm{cm}^{-1}\right)$ \\
\hline H & 921 (10858) & 54.8 (669) & 943 (10601) & $56.1(670)$ & 1075 (9301) & 115.5 (1201) & $22(257)$ \\
\hline 0 & 956 (10460) & $66.3(771)$ & 976 (10247) & 56.5 (587) & 1115 (8971) & 134.2 (1449) & $20(214)$ \\
\hline
\end{tabular}

The high photostability of these dyes and the brightness of their NIR fluorescence were demonstrated via stand-off imaging experiments with a home-built setup. In order to be properly imaged at a macroscopic setup, fluorophores need to be remarkably bright, considering that only a small fraction of emitted photons is able to reach the detector. Indeed, both $\mathrm{H}$ and $O$ showed similar fluorescence intensities, which were easily observable already with low integration times, low excitation powers in the Vis (LED lamp, no laser), and both at high and low concentrations (Figure S3, Figure 2a). In order to show their applicability as ink (e.g. for security ink technologies), letters were written on filter paper. Both $\mathrm{O}$ and $\mathrm{H}$ were clearly observable in the NIR channel, while not detectable in the visible (Figure 2b). When imaging these samples, an efficient cut-off of emission wavelengths was obtained with long pass filters (Figure 2c). Despite the $3 x$ lower absolute intensity at $\lambda_{\mathrm{em}}>1100 \mathrm{~nm}$, both $\mathrm{O}$ and $\mathrm{H}$ were still easily imaged (the slightly higher intensity of $O$ likely arises from 
local ink concentration differences, Figure 2d). These experiments show that both $\mathrm{O}$ and $\mathrm{H}$ dye can be used for imaging over a large range of the desired NIR spectral range. This finding is important because so far existing dyes such as ICG have been used for imaging far away from their emission maximum to get further into the NIR ${ }^{40}$.

In order to enhance their typically lower QYs as well as to overcome their typically limited water solubility, (NIR) organic dyes are often loaded into nano-/micrometer-sized polymeric microbeads. For this purpose, particles of different materials are commercially available. Among them, polystyrene (PS) beads appear appealing for in vivo imaging thanks to their mechanical stability, biocompatibility, robust synthetic protocols (miniemulsion polymerization) and broad range of sizes with narrow size distributions. Furthermore, diverse surface functionalizations are possible ${ }^{41}$. In terms of fluorophore incorporation techniques, the staining of particles via swelling represents one of the simplest options with regards to experimental complexity, yields and costs. Besides swelling, several PS loading protocols exist ${ }^{42}$, but BODIPY incorporation requires different approaches. The main challenge was to solubilize the BODIPY dye, without dissolving the PS beads during encapsulation (see Experimental Section). a

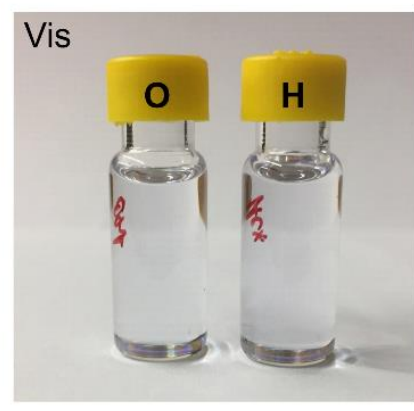

C

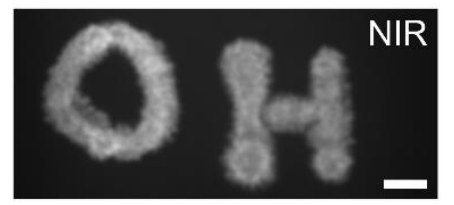

$\lambda_{\mathrm{em}}>900 \mathrm{~nm}$

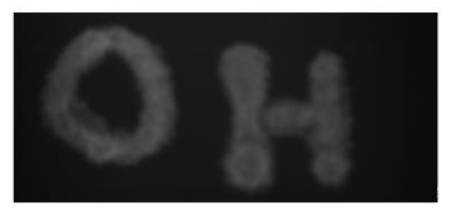

$\lambda_{\mathrm{em}}>1050 \mathrm{~nm}$
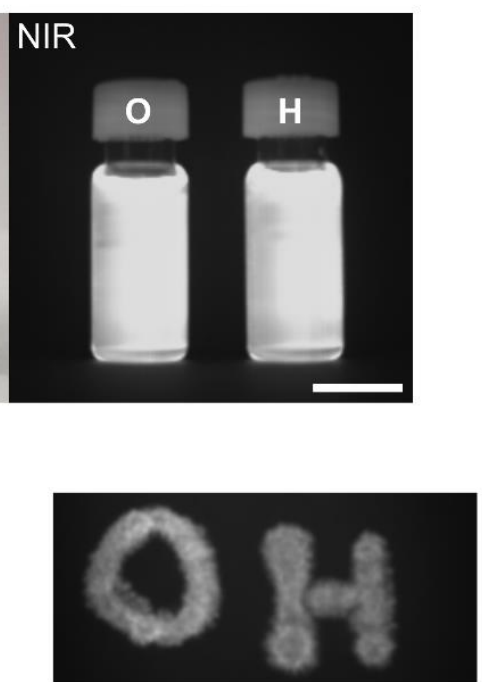

$\lambda_{\mathrm{em}}>950 \mathrm{~nm}$

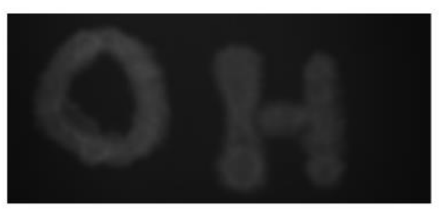

$\lambda_{\mathrm{em}}>1100 \mathrm{~nm}$ b

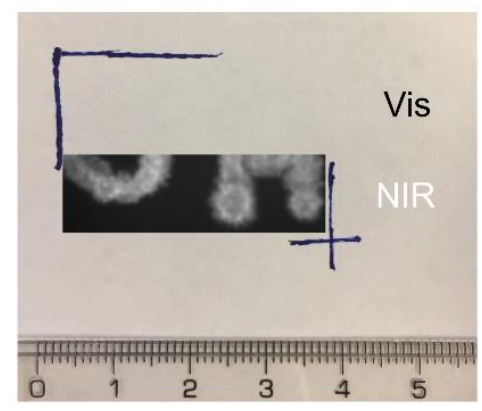

d

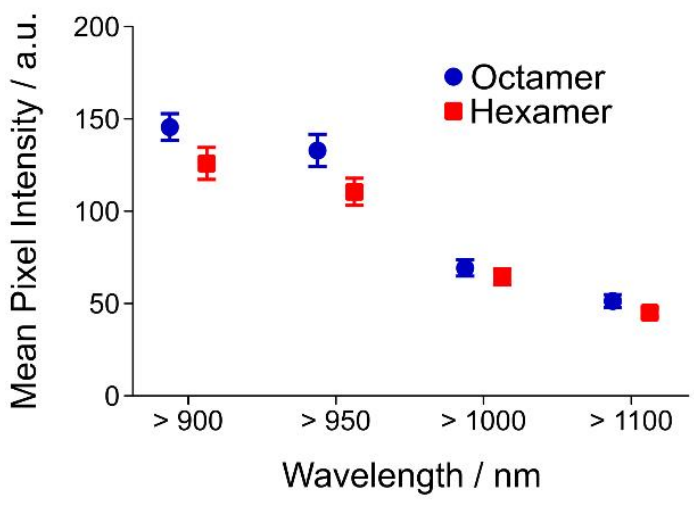

Figure 2: Remote NIR fluorescence imaging of BODIPYs. a Visible and corresponding NIR fluorescence picture of the octamer-BODIPY (O) and the hexamer-BODIPY (H) in toluene. Scale bar $=1 \mathrm{~cm}$. Exposure time $500 \mathrm{~ms}$. b BODIPY dye print on a filter paper with overlaid NIR fluorescence. The letters are only detectable in the NIR (scale in $\mathrm{cm}$ ). $\mathbf{c}$ NIR images of the letters for different long pass emission filters $\left(\lambda_{\text {em }}\right)$. Scale bar $=0.5 \mathrm{~cm}$. Exposure time $=500 \mathrm{~ms}$. $\mathrm{d}$ Relative signal of the two letters written in NIR at different wavelengths. The values are mean pixel intensities, evaluated from the pictures in c. Note that, even though the intensity decreases, there can be advantages such as less scattering or autofluorescence for using only the longer wavelengths. Error bars = standard deviation (from 5 different regions of interest within the dye print). $N=1$ independent sample.

According to the images acquired at our NIR microscopic imaging setup, the coating procedure was successful: the PS particles indeed displayed a bright and uniform fluorescence with neglectable leakage and remarkable photostability (Figure 3a, Figure S4). Furthermore, a similar staining protocol performed on silica beads also yielded homogeneously coated particles, thus highlighting the versatility of these oligo-BODIPYs (Figure S5).

Next, we introduced the hexamer-labelled PS beads into actin networks. The globular protein actin is the most abundant cytoskeletal protein in most eukaryotic cells and highly important for structure and function. The mechanical (viscoelastic) properties of actin networks are therefore an active field of study, and tools that measure these properties are thus of high interest. The self-assembly of actin monomers can be easily tailored in vitro by e.g. the employment of appropriate buffers and cross-linking proteins, thus allowing for an extensive amount of studies on actin filaments and networks ${ }^{43,44}$. In order to perform co-localization measurements, such actin filaments can be labelled with e.g. visible fluorophores: in our case, bundled filaments of actin were labelled with a green-emitting dye. Thanks to the 
brightness of our oligo-BODIPYs as well as to the lack of autofluorescence in the NIR, the dye-labelled PS beads were still clearly distinguishable after introduction into bundled actin networks (Figure $\mathbf{3 b}$ ). Next, the robust (photo)stability of $\mathrm{H}$ allowed us to exploit its fluorescence signal to perform passive microrheology in the form of video particle tracking (VPT) of the beads (Figure 3c). With this technique, which relies on the Generalized Stokes-Einstein relation, one can evaluate the viscoelastic properties of a system (i.e. our actin network) via the Fluctuation Dissipation Theorem. a

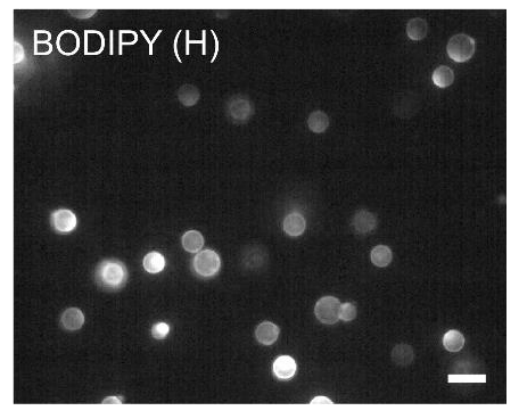

b
C

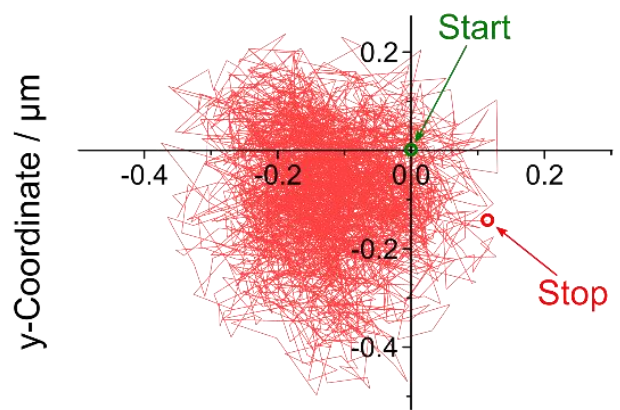

$\mathrm{x}$-Coordinate / $\mu \mathrm{m}$ d

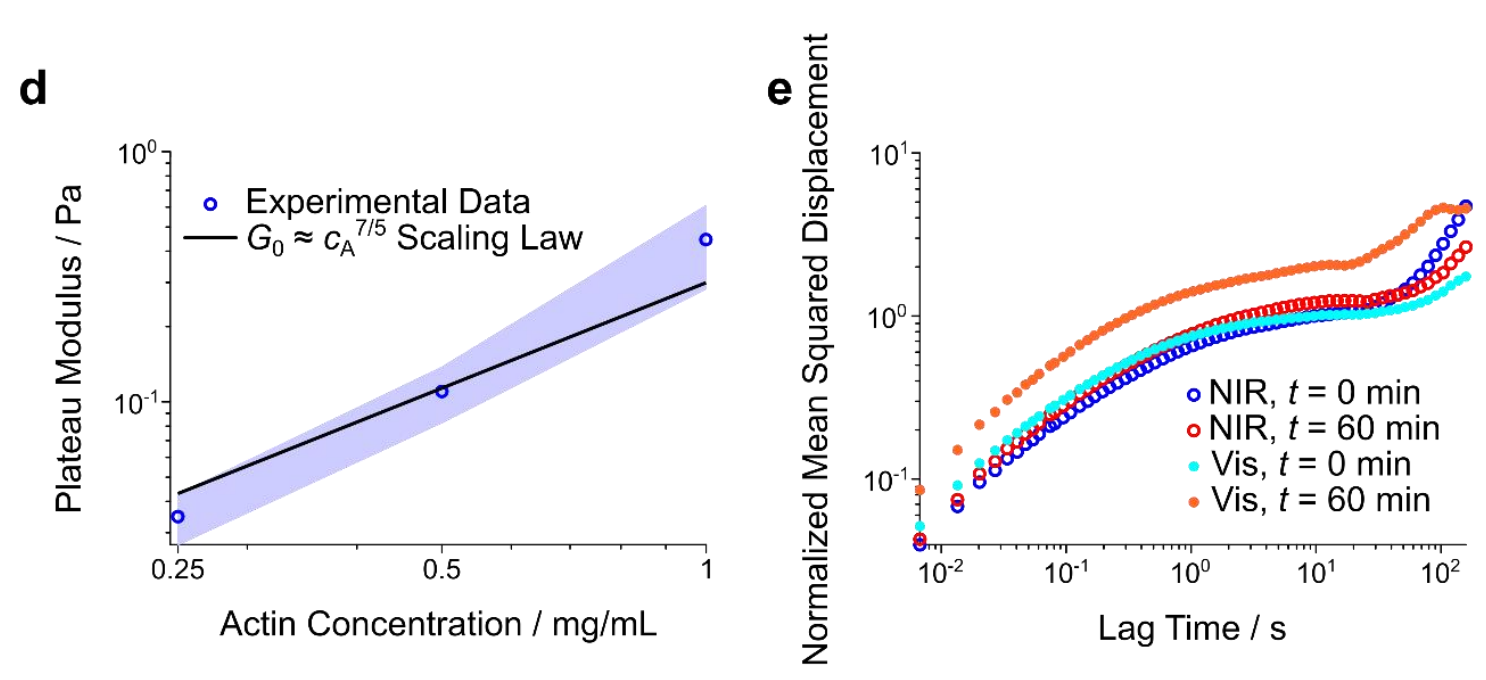

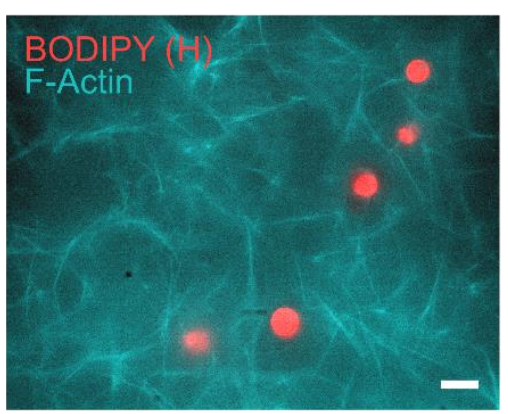

Figure 3: NIR microrheology in actin biopolymer networks. a NIR fluorescence image of polystyrene (PS) beads, which were coated with the NIR dye (hexamer). Scale bar = $10 \mu \mathrm{m}$. b NIR/Vis fluorescence image of dye-coated PS beads (red) inside a F-actin network (cyan). In order to allow co-localization, the actin filaments were stained with a Vis dye. Scale bar $=10 \mu \mathrm{m}$. c An exemplary trajectory obtained after video particle tracking (VPT) of NIR dye-coated PS beads in actin networks. The initial and final bead positions are indicated by the arrows. $\mathbf{d}$ Mechanical properties of actin networks are extracted from trajectories obtained by NIR microrheology. Here, the dependence of actin concentration $\left(c_{\mathrm{A}}\right)$ on plateau modulus $\left(G_{0}\right)$ is shown. The mean values (blue circles) and the corresponding standard deviations (light blue band) are plotted for the tested concentrations. The shear modulus of $G_{0}$ of such viscoelastic networks is known to scale as $G_{0} \approx c_{A}{ }^{7 / 5}$ (black line) ${ }^{44} . N=3$ independent samples. $n=11,27,31$ beads for $c_{A}=0.25,0.5$ and $1 \mathrm{mg} / \mathrm{mL}$, respectively. e Higher stability of actin networks using NIR-VPT. Mean squared displacement (MSD) curves obtained from NIR-VPT of PS beads in actin samples during prolonged continuous excitation. The results show that, using the NIR approach, the networks remain stable over time, which is not the case for Vis-VPT. The beads were coated in 2 different ways: either with our hexamer NIR dye (NIR) or with a visible dye (Vis, i.e. fluorescent red PS microspheres, 545/566 nm). The datasets were normalized to the respective $t=0$ min MSD at lag time $=10 \mathrm{~s}$ (i.e. in the middle of the plateau region). NIR dataset: $n=114$ beads at $t=0 \min$ and 169 beads at $t=60 \min$. Vis dataset: $n=103$ beads at $t=0$ min and 184 beads at $t=60 \min$. $N=5$ independent samples.

The Brownian motion of the incorporated probe particles (spheroidal microparticles, i.e. our beads) was quantified as mean squared displacement (MSD) and translated into shear moduli, which allowed a comparison of mechanical properties ${ }^{45}$. One open question is how actin concentration affects mechanical properties and this question can be answered by microrheology. Therefore, we measured the plateau modulus $\left(G_{0}\right)$, i.e. the value of the storage modulus at the frequency for which the ratio loss/storage moduli $(\delta)$ is minimum, for three different actin concentrations. The obtained values followed a scaling law predicted via the Tube Model and validated experimentally for such systems ${ }^{44}$ (Figure 3d). Finally, the impact of different coatings of PS beads on the degradation of actin networks was studied. It is known that illumination of Vis fluorescent (PS) beads can lead to drastic softening of actin gels, likely caused by generation of free oxygen radicals and photodamage of actin ${ }^{46,47}$. While this mechanism might be on one side used for disintegrationtriggered manipulation of actin networks, it also poses a threat and bias to most microrheological studies. With this motivation, 
we compared PS beads of same size with two different coatings: our $\mathrm{H}$ dye and a commercially available Vis control (i.e. fluorescent red PS microspheres, 545/566 nm, FRP5000, Lab 261). VPT analysis was performed with the camera and tracking software of an acoustic force spectroscope (AFS). In this way, MSDs could be measured at two different times: $t=0 \mathrm{~min}$ and $t=60 \mathrm{~min}$, with continuous illumination. Our dataset displayed a clear trend: the Vis beads showed the largest increase of mean squared displacements over time, indicating a higher degree of actin softening (Figure 3e, Figure S6-S7). It is thus plausible that the triplet states normally responsible for generation of toxic oxygen species are not accessible or were not created around the $\mathrm{H}$ dye. These results therefore highlight the advantage of using such versatile and robust NIR dyes for microrheology in polymeric networks and biological systems.

\section{Conclusions}

We report for the first time the NIR fluorescence of hexameric (H) and octameric (O) benzene-fused oligo-BODIPYs. Their broad excitation range and their narrow emission maxima between 940-1120 nm were studied via 1D and 2D spectroscopy. In different organic solvents, solvatochromic shifts appeared, whereas red-shifted emission wavelengths and peak sharpening trends were detected at higher concentrations, indicating the presence of J-aggregates. The NIR fluorescence of both $\mathrm{H}$ and $\mathrm{O}$ proved to be bright enough to enable stand-off detection and showing that these dyes can be used e.g. as security inks, for barcoding or for remote biomedical imaging. Furthermore, we demonstrated the potential of this class of BODIPYs as NIR fluorophores by coating them on silica and polystyrene microbeads. The latter could be used for NIR fluorescent video particle tracking (VPT) in biopolymer (actin) networks, without prominent actin degradation, a phenomenon occurring with conventional microrheological fluorophores.

In conclusion, thanks to their straightforward synthesis and advantageous photophysical properties, these oligo-BODIPYs are powerful tools for NIR bioimaging and photonics in general.

\section{Experimental}

\section{UV-Vis and NIR spectroscopy}

Absorption spectroscopy was performed with a JASCO V-670 device from 400 to $1100 \mathrm{~nm}$ in $0.2 \mathrm{~nm}$ steps in a $10 \mathrm{~mm}$ path quartz cuvette, using $a \sim 0.5 \mu \mathrm{M}$ dye solution $\left(A_{\max } \approx 0.2\right)$ in various solvents. The same dye solutions were transferred into glass vials and used for NIR fluorescence spectroscopy measurements, which were acquired with a Shamrock 193i spectrometer (Andor Solis Software, version 4.29.30012.0, Andor Technology Ltd.) connected to an IX53 microscope (Olympus). Excitation was performed with a gem 561 laser (Laser Quantum), a Cobolt Jive laser (Cobolt AB) and a monochromator MSH150, equipped with a LSE341 light source (LOT-Quantum Design GmbH). Typical parameters for NIR fluorescence measurements were $50 \mathrm{~mW}(\lambda=561 \mathrm{~nm})$ laser intensity, $200 \mu \mathrm{m}$ spectrometer slit width and $1 \mathrm{~s}$ integration time, using a center wavelength of $1000 \mathrm{~nm}$ with a spectral grating of $150 \mathrm{l} / \mathrm{mm}$.

A self-written Python code was used to plot the 2D spectra, which were not corrected for the spectral irradiance of the xenon lamp. Further data analysis and plotting were performed in Origin Pro 8.1.

\section{NIR camera-assisted stand-off detection}

NIR stand-off detection was performed with a custom made, portable setup, using a XEVA (Xenics, Leuven Belgium) NIR InGaAs camera (Kowa objective, $f=25 \mathrm{~mm} / \mathrm{F} 1.4$ ) and a whitelight source (UHPLCC-01, UHP-LED-white, Prizmatix) equipped with a $700 \mathrm{~nm}$ short pass filter (FESH0700, Thorlabs) for excitation. Optical filters (FEL0900, FEL0950, FEL1050, FEL1100, ThorLabs) in a manual filter wheel (CFW6/M, Thorlabs) were mounted in front of the camera, which was equipped with an additional $900 \mathrm{~nm}$ long pass filter (FEL0900, Thorlabs). Stand-off distance for NIR fluorescence detection was $20 \mathrm{~cm}$. For imaging of NIR dyes in vials, the following settings were chosen: integration time $=0.5 \mathrm{~s}$, light intensity $=20 \mathrm{~mW} / \mathrm{cm}^{2}$. Parameters for NIR ink samples were instead: integration time $=0.5 \mathrm{~s}$, light intensity $=35 \mathrm{~mW} / \mathrm{cm}^{2}$. Concentration of dyes in vials were $\approx 4 \mu \mathrm{M}$ for the octamer and $\approx 5 \mu \mathrm{M}$ for the hexamer solution in toluene. Approximately $10 \mu \mathrm{L}$ of these solutions were drawn on standard filter paper to simulate the NIR ink. Light intensity was measured at $570 \mathrm{~nm}$ with a power meter (PM16-121, Thorlabs).

Data analysis was performed in ImageJ (v. 1.52a), whereas plotting was carried out in Origin Pro 8.1.

\section{Coating of microbeads}

The coating of polystyrene (PS) and silica $\left(\mathrm{SiO}_{2}\right)$ microspheres were performed as followed.

The following PS beads were used: Polybead ${ }^{\circledR}$ Microspheres, $d \approx 1.5 \mu \mathrm{m}$, Polysciences for rheology of different actin concentrations; PST5000, $d \approx 5 \mu \mathrm{m}$, Lab 261 for the actin degradation dataset. First, $300 \mu \mathrm{L}$ of aqueous suspension of plain PS beads were removed from the original flask and directly mixed in a glass snap cap vial with $300 \mu \mathrm{L}$ of methanol. The vial was placed on a heating plate at $\approx 50^{\circ} \mathrm{C}$ and was kept under $\mathrm{N}_{2}$ flow during all preparation steps to speed up the drying process. As soon as the solvents had evaporated, other $400 \mu \mathrm{L}$ of methanol were introduced. After evaporation, other $300 \mu \mathrm{L}$ of methanol were introduced and sonicated for $\approx 1 \mathrm{~min}$. Immediately afterwards $300 \mu \mathrm{L}(\approx 5 \mu \mathrm{M})$ of the hexamer dye $(\mathrm{H})$ dissolved in diethyl ether was added to the PS beads-methanol suspension, mixed and directly placed for evaporation under $\mathrm{N}_{2}$ stream. Finally, the dried beads were dispersed in $600 \mu \mathrm{L}$ of milliQ water, and the system was bath sonicated for $\approx 2$ min. The so-prepared H-coated PS microspheres were transferred into brown snap cap vials (to reduce the chances of photobleaching) and were stored at $4{ }^{\circ} \mathrm{C}$.

With the previously described setup, plain $\mathrm{SiO}_{2}$ microspheres (SS06N, $d \approx 6 \mu \mathrm{m}$, Bangs Laboratories, Inc.) were coated in a similar way. To start with, $300 \mu \mathrm{L}$ of aqueous suspension of 
beads were removed from the original flask and directly mixed with $1 \mathrm{~mL}$ of acetonitrile. After drying, another $1 \mathrm{~mL}$ of acetonitrile was added. As soon as evaporation was complete, $1 \mathrm{~mL}$ of toluene and $300 \mu \mathrm{L}(\approx 5 \mu \mathrm{M})$ of $\mathrm{H}$ dissolved in toluene were introduced into the glass vial. After evaporation, addition of another $1 \mathrm{~mL}$ of toluene and another drying step, the $\mathrm{SiO}_{2}$ microspheres were dispersed in $1 \mathrm{~mL}$ of milliQ water and bath sonicated for $\approx 2 \mathrm{~min}$. The so-prepared $\mathrm{H}$-coated $\mathrm{SiO}_{2}$ microspheres were transferred into brown snap cap vials (to reduce the chances of photobleaching) and were stored at $4{ }^{\circ} \mathrm{C}$.

\section{Preparation of Actin Networks}

Actin (rabbit skeletal muscle, Cytoskeleton) networks were assembled at different concentrations: $0.25,0.5$ and $1 \mathrm{mg} / \mathrm{mL}$ for VPT verification, $1 \mathrm{mg} / \mathrm{mL}$ for degradation experiments, and $1.8 \mathrm{mg} / \mathrm{mL}$ for microscopy images of bundled actin. The chemicals listed below, unless explicitly mentioned, were purchased from Sigma-Aldrich.

First, the lyophilized actin powder was dissolved in ultrapure water (to $10 \mathrm{mg} / \mathrm{mL}$ ). Next, the actin was introduced into a stabilizing buffer ( $5 \mathrm{mM}$ Tris- $\mathrm{HCl} \mathrm{pH} 8.0,0.2 \mathrm{mM} \mathrm{CaCl}_{2}, 0.2 \mathrm{mM}$ ATP, $5 \%$ sucrose, and $1 \%$ dextran). The system was then further diluted with freshly prepared G-buffer $(2 \mathrm{mM}$ Tris/ $\mathrm{HCl}, 0.1 \mathrm{mM}$ $\mathrm{CaCl}_{2}, 0.2 \mathrm{mM}$ ATP, $0.5 \mathrm{mM}$ dithiothreitol, $0.1 \mathrm{mM} \mathrm{NaN}_{3}, \mathrm{pH} 7.8$ ) and depolymerized (30 $\mathrm{min}$ on ice). Microparticles were first washed via centrifugation, i.e. the supernatant was removed and the system refilled with G-buffer $\approx 3-5$ times. Then, the beads were added directly before the polymerization step. Similarly, for the bundled networks Alexa Fluor 488-phalloidin (Thermo Fischer) was added in this step for co-localization. The polymerization was started by adding $25 \%$ of the total volume in F-buffer ( $500 \mathrm{mM} \mathrm{KCl}, 20 \mathrm{mM} \mathrm{MgCl} 2,50 \mathrm{mM}$ EGTA, $0.1 \mathrm{mM}$ $\mathrm{NaN}_{3}, \mathrm{pH}$ 7.4) and vortexing. The samples were carefully but quickly transferred into glass chambers (i.e. cleaned \#1 cover slips connected by $130 \mu \mathrm{m}$ cut out double sided tape) and left to fully polymerize and equilibrate overnight at room temperature.

\section{NIR microscopic imaging}

Our home-built NIR imaging setup consists of a $561 \mathrm{~nm}$ laser (Cobolt JiveTM $561 \mathrm{~nm}$ ), a fluorescence lamp (X-Cite ${ }^{\circ}$ 120Q, Excelitas Technologies), Olympus IX53 microscope equipped with a 100x (UPlanSApo 100x/1.35 Sil, Olympus) objective, a NIR camera (Cheetah TE1, Xenics) and a Vis camera (Zyla 5.5 sCMOS, Oxford Instruments). For NIR imaging, the laser was used as excitation source, whereas a dichroic mirror (Vis/NIR, HC BS R785 lambda/5 PV, F38-785S, AHF) and a $900 \mathrm{~nm}$ long-pass filter (FELH0900, Thorlabs) were installed along the light path to the NIR camera. For Vis imaging at GFP wavelengths with the Zyla camera, the fluorescence lamp was employed for excitation at maximum power, and the following filters were used: ET470/40x (Set 49002, Chroma) for excitation, HC BS R488 1 lambda PV flat (F38-489, AHF) as dichroic mirror in the microscope's filter cube, the previously mentioned Vis/NIR dichroic mirror, a laser/Vis dichroic mirror (T550LPXR, $25.5 \times 36 \times 3 \mathrm{~mm}$, Chroma), and finally ET525/50m (Set 49002, Chroma), a Notch 561 (zet561NF F40-562, AHF) and a short-pass filter (650/SP HC, F37-650, AHF) directly mounted in front of the Vis camera.

For qualitative images of coated PS microspheres, an aliquot of bead suspension was diluted (typically, 1:10 in volume) with water. Then, a small volume (typically $10 \mu \mathrm{L}$ ) was removed from the diluted suspension and simply drop-casted on a \#1 glass coverslip placed above the objective. Afterwards, imaging of this droplet was performed either in wet (Fig. 3a) or dried (Fig. S4) form according to the following parameters: set laser power $=250 \mathrm{~mW}$, exposure time of NIR camera $=0.1 \mathrm{~s}$.

When beads were embedded in actin networks, the previously described (GFP-optimized) filters allowed the detection of the Alexa Fluor 488-labelled actin filaments. For qualitative observation of $\mathrm{PS}$ and $\mathrm{SiO}_{2}$ beads embedded in ion-bundled actin networks, images were taken as follows: set laser power $=250 \mathrm{~mW}$, exposure time of NIR camera $=1 \mathrm{~s}$, exposure time of Vis camera $=0.02-0.03 \mathrm{~s}$.

The plateau moduli of actin samples of 3 different concentrations were measured by tracking the NIR fluorescent beads over time. In this way, only the NIR camera was employed for data acquisition, which was carried out with these settings: set laser power $=250 \mathrm{~mW}$, exposure time $=0.05 \mathrm{~s}$, frame rate $=19 \mathrm{fps}$, total measurement time $=3 \mathrm{~min}$.

Characterization images of dye-coated beads drop-casted on glass or embedded in actin networks were elaborated in Image (v. 1.52a).

\section{Bright field microscopy}

Passive microrheology measurements for actin degradation experiments were performed using a bright field microscope (Lumicks, AFS) at a high frame rate (138 fps) with a 60x objective (CFI Achro-mat FF, WD $=0.3 \mathrm{~mm}, \mathrm{NA}=0.80$, Nikon). Tracking of the microparticles was done with the AFS instrument software (Lumicks), which uses a quadrant algorithm to track ring shaped diffraction patterns in two dimensions. To avoid electrostatic interaction with the glass surfaces, the focal plane was set at heights not lower than $20 \mu \mathrm{m}$. Between the starting ( $t=0 \mathrm{~min}$ ) and final ( $t=60 \mathrm{~min}$ ) measurement sessions, samples were placed for continuous excitation under a fluorescent lamp (PhotoFluor II NIR, AHF) installed on an Olympus BX51 microscope. This lamp was employed at maximum intensity $\left(\lambda_{\text {exc }}=545 \mathrm{~nm}\right)$ and the light was directed to the samples through a 10x objective (MPlanFL N, Olympus) with a measured output power on the sample plane corresponding to $\approx 83 \mathrm{~mW}$.

\section{Microrheology analysis}

For the VPT of PS beads in actin networks of different concentrations, the raw images were first despeckled and their contrast was enhanced in ImageJ (v. 1.52a). Next, a custommade Python ${ }^{48}$ code based on the Trackpy ${ }^{49,50}$ package (Python v. 3.7.7, Trackpy v. 0.5.0) was used to obtain the trajectories. Typically chosen parameters for the tracking algorithm were: blob diameter $=31$ pixels, memory $=10$ frames, maximum displacement between consecutive frames $=25$ pixels, minimum trajectory length $=3400$ frames (out of 3400 total frames). 
Trajectories obtained from both actin datasets (i.e. different actin concentrations and actin degradation) were analyzed based on the work of Crocker et. $\left.a\right|^{51}$ with a customized variant of a MATLAB script made freely available on UMass (people.umass.edu/kilfoil) by Maria Kilfoil.

\section{Conflicts of interest}

There are no conflicts to declare.

\section{Acknowledgements}

Funded by the Deutsche Forschungsgemeinschaft (DFG, German Research Foundation) under Germany's Excellence Strategy-EXC 2033-390677874-RESOLV. We acknowledge support by the DFG via the Heisenberg program (S.K.).

\section{References}

G. Hong, A. L. Antaris and H. Dai, Nat. Biomed. Eng., 2017, 1, 0010.

S. Kruss, A. J. Hilmer, J. Zhang, N. F. Reuel, B. Mu and M. S. Strano, Adv. Drug Deliv. Rev., 2013, 65, 1933-1950. A. Smith, M. C. Mancini and S. Nie, Nat. Nanotechnol. 2009, 4, 710-711.

4 E. A. Owens, M. Henary, G. El Fakhri and H. S. Choi, Acc. Chem. Res., 2016, 49, 1731-1740. J. Cao, B. Zhu, K. Zheng, S. He, L. Meng, J. Song and H. Yang, Front. Bioeng. Biotechnol., 2020, 7, 1-21. P. Liu, X. Mu, X. D. Zhang and D. Ming, Bioconjug. Chem. 2020, 31, 260-275.

7 A. Spreinat, G. Selvaggio, L. Erpenbeck and S. Kruss, J. Biophotonics, , DOI:10.1002/jbio.201960080. A. J. Gillen and A. A. Boghossian, Front. Chem., 2019, 7, 113.

M. Dinarvand, S. Elizarova, J. Daniel and S. Kruss, Chempluschem, 2020, 85, 1465-1480.

10 M. Zheng, Top. Curr. Chem., 2017, 375, 1-36.

11 R. Nißler, L. Kurth, H. Li, A. Spreinat, I. Kuhlemann, B. S. Flavel and S. Kruss, Anal. Chem., 2021, 93, 6446-6455. S. M. Bachilo, M. S. Strano, C. Kittrell, R. H. Hauge, R. E. Smalley and R. B. Weisman, Science (80-. )., 2002, 298, 2361-2366. H. Wu, R. Nißler, V. Morris, N. Herrmann, P. Hu, S. Jeon, S. Kruss and J. P. Giraldo, Nano Lett., 2020, 20, 2432-2442. R. Nißler, O. Bader, M. Dohmen, S. G. Walter, C. Noll, G. Selvaggio, U. Groß and S. Kruss, Nat. Commun., 2020, 11, 1-12.

15 M. Dinarvand, E. Neubert, D. Meyer, G. Selvaggio, F. A. Mann, L. Erpenbeck and S. Kruss, Nano Lett., 2019, 19, 6604-6611.

16 S. Kruss, D. P. Salem, L. Vuković, B. Lima, E. Vander Ende, E. S. Boyden and M. S. Strano, Proc. Natl. Acad. Sci., 2017, 114, 1789-1794. Chem. Rev., 2020, 120, 2693-2758.

M. Prato, K. Kostarelos and A. Bianco, Acc. Chem. Res., 2008, 41, 275-294.

A. H. Brozena, M. Kim, L. R. Powell and Y. H. Wang, Nat. Rev. Chem., 2019, 3, 375-392.

F. A. Mann, N. Herrmann, F. Opazo and S. Kruss, Angew. Chemie Int. Ed., 2020, 59, 17732-17738.

A. Spreinat, M. M. Dohmen, J. Lüttgens, N. Herrmann, L. F. Klepzig, R. Nißler, S. Weber, F. A. Mann, J. Lauth and S. Kruss, J. Phys. Chem. C, 2021, 125, 18341-18351. G. Selvaggio, A. Chizhik, R. Nißler, L. Kuhlemann, D. Meyer, L. Vuong, H. Preiß, N. Herrmann, F. A. Mann, Z. Lv, T. A. Oswald, A. Spreinat, L. Erpenbeck, J. Großhans, V. Karius, A. Janshoff, J. Pablo Giraldo and S. Kruss, Nat. Commun., 2020, 11, 1495.

G. Selvaggio, M. Weitzel, N. Oleksiievets, T. A. Oswald, R. Nißler, I. Mey, V. Karius, J. Enderlein, R. Tsukanov and S. Kruss, Nanoscale Adv., , DOI:10.1039/D1NA00238D.

A. Mishra, R. K. Behera, P. K. Behera, B. K. Mishra and G. B. Behera, Chem. Rev., 2000, 100, 1973-2011.

L. Feng, W. Chen, X. Ma, S. H. Liu and J. Yin, Org. Biomol. Chem., 2020, 18, 9385-9397.

L. Hu, Z. Yan and H. Xu, RSC Adv., 2013, 3, 7667-7676. C. Lambert, T. Scherpf, H. Ceymann, A. Schmiedel and M. Holzapfel, J. Am. Chem. Soc., 2015, 137, 3547-3557. M. Kaur and D. H. Choi, Chem. Soc. Rev., 2015, 44, 58-77. G. M. Fischer, A. P. Ehlers, A. Zumbusch and E. Daltrozzo, Angew. Chemie - Int. Ed., 2007, 46, 3750-3753.

A. Loudet and K. Burgess, Chem. Rev., 2007, 107, 48914932.

G. Ulrich, R. Ziessel and A. Harriman, Angew. Chemie - Int. Ed., 2008, 47, 1184-1201.

J. Killoran, L. Allen, J. F. Gallagher, W. M. Gallagher and D. F. O'Shea, Chem. Commun., 2002, 317, 1862-1863.

A. Kamkaew and K. Burgess, Chem. Commun., 2015, 51, 10664-10667.

L. J. Patalag, P. G. Jones and D. B. Werz, Angew. Chemie Int. Ed., 2016, 55, 13340-13344.

L. J. Patalag, M. Loch, P. G. Jones and D. B. Werz, J. Org. Chem., 2019, 84, 7804-7814.

K. Umezawa, A. Matsui, Y. Nakamura, D. Citterio and K. Suzuki, Chem. - A Eur. J., 2009, 15, 1096-1106. K. Rurack, M. Kollmannsberger and J. Daub, Angew. Chemie - Int. Ed., 2001, 40, 385-387.

A. Patra, L. J. Patalag, P. G. Jones and D. B. Werz, Angew. Chemie - Int. Ed., 2021, 60, 747-752.

L. J. Patalag, L. P. Ho, P. G. Jones and D. B. Werz, J. Am. Chem. Soc., 2017, 139, 15104-15113.

J. A. Carr, D. Franke, J. R. Caram, C. F. Perkinson, M. Saif, V. Askoxylakis, M. Datta, D. Fukumura, R. K. Jain, M. G. Bawendi and O. T. Bruns, Proc. Natl. Acad. Sci. U. S. A., 2018, 115, 4465-4470.

M. A. Markus, J. Napp, T. Behnke, M. Mitkovski, S. Monecke, C. Dullin, S. Kilfeather, R. Dressel, U. ReschGenger and F. Alves, ACS Nano, 2015, 9, 11642-11657. T. Behnke, C. Würth, E. M. Laux, K. Hoffmann and U. ReschGenger, Dye. Pigment., 2012, 94, 247-257.

H. Isambert and A. C. Maggs, Macromolecules, 1996, 29 , 
1036-1040.

B. Hinner, M. Tempel, E. Sackmann, K. Kroy and E. Frey, Phys. Rev. Lett., 1998, 81, 2614-2617.

45 M. Tassieri, R. M. L. Evans, L. Barbu-Tudoran, G. N. Khaname, J. Trinick and T. A. Waigh, Phys. Rev. Lett., 2008, 101, 7-10.

T. Golde, C. Schuldt, J. Schnauß, D. Strehle, M. Glaser and J. Käs, Phys. Rev. E - Stat. Nonlinear, Soft Matter Phys., , DOI:10.1103/PhysRevE.88.044601.

47 S. Van De Linde, I. Krstić, T. Prisner, S. Doose, M. Heilemann and M. Sauer, Photochem. Photobiol. Sci., 2011, 10, 499-506. Python, Welcome to Python.org, https://www.python.org/, (accessed 26 November 2019). J. C. Crocker and D. G. Grier, J. Colloid Interface Sci., 1996, 179, 298-310.

50 D. Allan, C. van der Wel, N. Keim, T. A. Caswell, D. Wieker, R. Verweij, C. Reid, Thierry, L. Grueter, K. Ramos, apiszcz, zoeith, R. W. Perry, F. Boulogne, P. Sinha, pfigliozzi, N. Bruot, L. Uieda, J. Katins, H. Mary and A. Ahmadia, 2019. Kaplan, A. G. Yodh and D. A. Weitz, Phys. Rev. Lett., 2000, 85, 888-891. 